\title{
Visceral adiposity is associated with worse urinary and sexual function recovery after radical prostatectomy: Results from a longitudinal cohort study
}

\author{
Tommaso Cai ${ }^{1,2}$, Andrea Cocci ${ }^{3}$, Fabrizio Di Maida ${ }^{3}$, Stefano Chiodini ${ }^{1}$, Francesco Ciarleglio ${ }^{4}$, \\ Lorenzo Giuseppe Luciani ${ }^{1}$, Giovanni Pedrotti ${ }^{5}$, Alessandro Palmieri ${ }^{6}$, Gianni Malossini ${ }^{1}$, \\ Michele Rizzo ${ }^{7}$, Giovanni Liguori ${ }^{7}$, Truls E. Bjerklund Johansen ${ }^{2,8,9}$ \\ ${ }^{1}$ Department of Urology, Santa Chiara Regional Hospital, Trento, Italy; \\ 2 Institute of Clinical Medicine, University of Oslo, Oslo, Norway; \\ ${ }^{3}$ Department of Urology, University of Florence, Florence, Italy; \\ ${ }^{4}$ Department of Surgery, Santa Chiara Regional Hospital, Trento, Italy; \\ ${ }^{5}$ Department of Anesthesiology, Santa Maria del Carmine Hospital, Rovereto, Italy; \\ ${ }^{6}$ Department of Urology, University Federico II, Naples, Italy; \\ ${ }^{7}$ Department of Urology, University of Trieste, Trieste, Italy; \\ ${ }^{8}$ Department of Urology, Oslo University Hospital, Oslo, Norway; \\ ${ }^{9}$ Institute of Clinical Medicine, University of Aarhus, Denmark.
}

\begin{abstract}
Summary Objective: A prospective longitudinal cohort study on the impact of anthropometric measures on the sexual function and continence recovery in patients treated with laparoscopic radical prostatectomy (LRP) is presented.

Material and methods: Anthropometric measures, International Index of Erectile Function (IIEF-5) and International Prostatic Symptoms Score questionnaires, were collected before surgery and at the end of follow-up period. All patients were assigned into the following groups: A) non-obese; B) non-obese with central adiposity; C) obese without central adiposity; D) obese with central adiposity. Urinary and sexual functions were the outcome measures.

Results: At the end of follow-up, in 29 patients with visceral adiposity (VA) the median IIEF-5 was 14 (IQR 7-18) while in 49 non-VA patients $(62.8 \%)$ was $22(I Q R ~ 17-24)(p<0.001)$.

Twenty-three patients (79.3\%) with VA reported complete continence, while 6 (20.7\%) used $\geq 2$ pads per day. Forty-eight patients (97.9\%) without VA reported complete continence. VA was confirmed as a strong independent predictor for worse continence (HR 3.67; 2.75-4.51 CI95\% $p=0.003)$ and sexual function recovery (HR: 4.51; 3.09-5.63 C195\% $p<0.001$ ). Conclusion: We truly believe obese with visceral adiposity patients with prostate cancer should receive detailed preoperative counseling before surgery, including higher risk of suboptimal functional outcomes.
\end{abstract}

KEY WORDS: Prostate cancer; Adiposity; Metabolic syndrome; Body mass index; Quality of life.

Submitted 17 May 2021; Accepted 3 July 2021

\section{INTRODUCTION}

Several treatment options are available for the management of localized prostate cancer (PCa). To date, more than $40 \%$ of PCa patients have radical prostatectomy (RP) for their definitive treatment (1-2). Quality of life after surgery is strictly related to continence and potency sphere (3). As such, apart from cancer control, functional outcomes have been widely explored in an endeavor to timely predict which patients may experience worse sexual and continence recovery (4-5). In the last few years, obesity has emerged has a clinical factor potentially influencing perioperative features. Indeed, several studies have reported evidence for obesity being independently associated with higher complication rates (6), as well as worse oncologic (7) and functional outcomes after surgery (8). However, we are still far from drawing definitive conclusions. To date, current literature on this issue has been critically influenced by several features: 1) most studies have defined body habitus using body mass index (BMI), whilst data on district adiposity parameters such as waist circumference (WC), subcutaneous and abdominal fat were poorly investigated; 2) a significant body of evidence still derives from open RP series. As such, reported findings may be not completely contemporary, being RP increasingly performed nowadays by laparoscopic or robot-assisted approach. To address this unmet need, we designed this longitudinal cohort study with a long-term follow-up period to better understand the impact of abdominal visceral adiposity (VA), WC and BMI on the recovery of sexual function and continence in patients with PCa treated with laparoscopic RP (LRP).

\section{MATERIALS AND METHODS}

Patients, dataset and study schedule

All patients affected by localized intermediate-risk prostate cancer and treated with laparoscopy radical prostatectomy at our Centre between January and December 2012, have been enrolled in this longitudinal cohort study. Clinical (including BMI and WC), instrumental, surgical, and

No conflict of interest declared. 
pathological features were recorded before enrolment. All surgical procedures were performed by a single highly trained laparoscopic surgeon (GM). In brief, all procedures have been performed by using an extraperitoneal 5trocar approach (9). The vesico-urethral anastomosis was made via 2 running sutures with 2-0 Monocryl according to the technique described by Van Velthoven (10).

All patients underwent oncological follow-up evaluations, in line with International Guidelines, for prostate cancer and with our previous studies $(9,11)$. After six months, one year after surgery and at each year follow-up evaluation, additionally to the standard biochemical and instrumental evaluations, all patients underwent specific questionnaires about quality of life and sexual function. The Figure 1 shows the study schedule. The median follow-up period was 86 months (82-95). The study was conducted in line with the STROBE statement (http://www.strobestatement.org) and in line with the Good Clinical Practice guidelines and the ethical principles laid down in the latest version of the Declaration of Helsinki.

\section{Inclusion and exclusion criteria}

We consider all patients affected by localized intermediate-risk prostate cancer, in line with the definition and criteria of D'Amico (12), and candidates for laparoscopy radical prostatectomy.

We excluded from the study patients who had a history of erectile disfunction, patients on PDE- 5 or $5 \alpha$-reductase inhibitors, patients with penile prosthesis implants. Patients affected by hypotestosteronemia and with other concomitant major diseases were excluded. Finally, all patients who require adjuvant hormonal therapy after surgery were also excluded.

\section{Body mass index and anthropometric measures}

At the enrolling time, the following anthropometric measures have been collected: height $(\mathrm{cm})$, weight $(\mathrm{kg})$, and waist circumference $(\mathrm{cm})$ measurement. BMI was calculated as weight in $\mathrm{kg}$ divided by squared height in meters $\left(\mathrm{kg} / \mathrm{m}^{2}\right)$. The waist circumference was measured using a standard measurement strip with the patients standing and breathing normally, at the midway between the lowest rib margin and iliac crest. In line with the National Cholesterol Educational Program Adult Treatment Panel III (NCEP: ATP III) (13), a cut-off of $102 \mathrm{~cm}$ for the waist circumference and of $30 \mathrm{~kg} / \mathrm{m}^{2}$ for the BMI has been considered. In line with De Nunzio et al. (14). patients were then categorized in 4 body habitus groups: a) non-obese (BMI $<30 \mathrm{~kg} / \mathrm{m}^{2}$ and $\mathrm{WC}<102 \mathrm{~cm}$ )

b) non-obese with central adiposity (BMI $<30 \mathrm{~kg} / \mathrm{m}^{2}$ and $\mathrm{WC} \geq 102 \mathrm{~cm}$ )

c) obese without central adiposity (BMI $>30 \mathrm{~kg} / \mathrm{m}^{2}$ and $<\mathrm{WC} 102 \mathrm{~cm}$ )

d) obese with central adiposity (BMI $\geq 30 \mathrm{~kg} / \mathrm{m}^{2}$ and WC $\geq 102 \mathrm{~cm}$ )

Even if some authors stated that visceral adiposity index was shown to be a better surrogate index than these single anthropometric indices to use in clinical practice, we decided to not use it due to the complexity of its calculation $(15,16)$. In fact, visceral adiposity index is comprised of anthropometric measures like BMI, WC and clinical measures of serum triglycerides and high-density lipoprotein-cholesterol levels (15).

\section{Data collection and urological evaluations at each follow-up visit}

At the time of surgery, in addition to all anthropometric measures, the following parameters were recorded: the patient's and partner's age, the Charlson comorbidity index, preoperative prostate-specific antigen levels, Gleason score, clinical prostate cancer stage (through an abdominal computed tomography (CT) scan and skeletal scintigraphy), duration of hospital stay and surgical complications. All patients underwent a standard follow-up schedule (Figure 1) depending on individual tumors and characteristics, in line with International Guidelines and in line with our everyday clinical practice $(1,17)$. In brief, clinical evaluation with DRE, prostate-specific antigen level and instrumental evaluation. Moreover, after six months, one year after surgery and at each year follow-up evaluation all patients underwent the following questionnaires: International Index of Erectile Function (IIEF-5) (18) and International Prostatic Symptoms Score (IPSS) (19) questionnaires, in line with previous study (20). Continent patients were defined by use of 0 or 1 safety pad/day (11).

\section{Outcome measures}

The main outcome measures were change in questionnaire score, the urinary and sexual function recovery at the end of the follow-up evaluation in each body habitus groups.

\section{Statistical analysis}

For statistical purposes, independent variables included all patient- and tumor-related data available in our institutional database. First, descriptive statistics were obtained reporting medians and interquartile range (IQR, $25^{\text {th }}$ and $75^{\text {th }}$ percentiles) for continuous variables, and frequencies and proportions for categorical variables, as appropriate.

Figure 1.

Follow-up schedule.

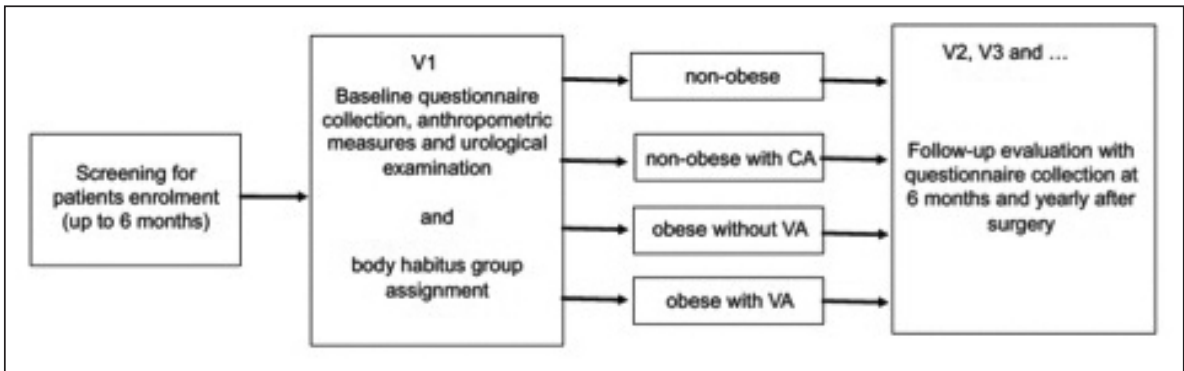

Continuous variables were compared using the Student t test. Categorical variables were tested with the chi-square test. BMI and waist circumference were examined as continuous variables using crude and adjusted logistic regressions to evaluate their association with the recov- 
Table 2.

Functional findings at the follow-up evaluation according to the body habitus.

\begin{tabular}{|c|c|c|c|c|}
\hline \multirow{2}{*}{$\begin{array}{l}\text { Patients }\left(\mathrm{n}^{\circ}\right) \\
\text { NCEP: ATP III\# (body habitus) }\end{array}$} & \multicolumn{4}{|c|}{78} \\
\hline & Group A & Group B & Group C & Group D \\
\hline Patients $\left(n^{\circ}\right)$ & $23(29.4)$ & $9(11.5)$ & $26(33.4)$ & $20(25.7)$ \\
\hline \multicolumn{5}{|l|}{ Urinary continence } \\
\hline No pad/die & 19 & 7 & 20 & 12 \\
\hline No or $1 \mathrm{pad} / \mathrm{die}$ & 4 & 1 & 5 & 3 \\
\hline 2 or more pads/die & 0 & 1 & 1 & 5 \\
\hline IIEF-5§ (median; IQR*) & $22(18-23)$ & $14(7-16)$ & $22(17-24)$ & $14(8-18)$ \\
\hline IPSS' & $11(6-14)$ & $12(7-13)$ & $10(6-11)$ & $11(6-13)$ \\
\hline
\end{tabular}

\section{Functional outcomes}

Overall, 71 (91\%) patients reported complete continence, while 7 (8.9\%) used $\geq 2$ pads per day. Median postoperative IIEF-5 was 18 (IQR: 7-24). Twelve patients (15.4\%)

Figure 3.

a) Median IIEF-5 in patients by body habitus at baseline and at the end of the study. b) Median IIEF-5 in patients with and without visceral adiposity at baseline and at the end of the study.

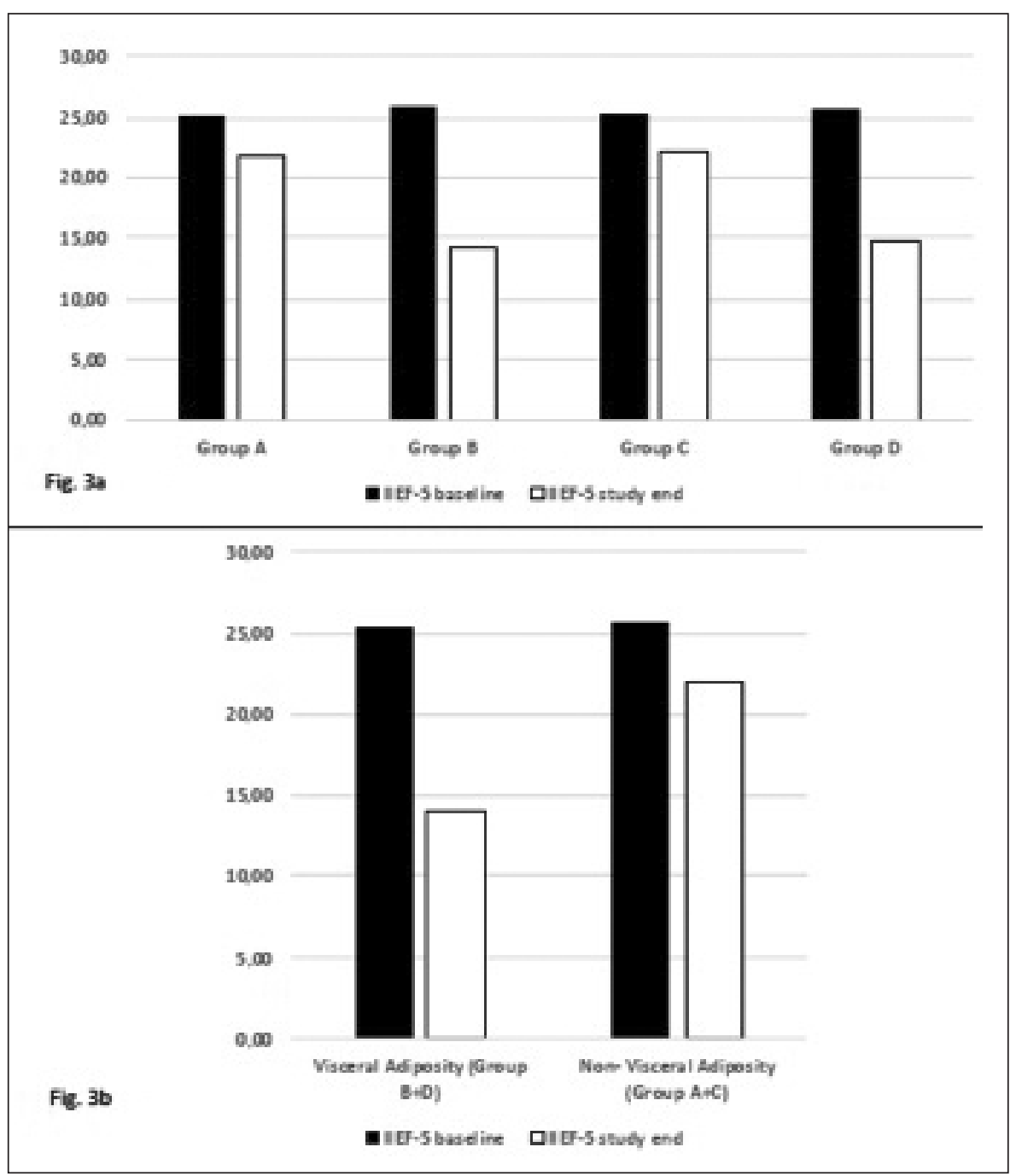

reported spontaneous erection without any pharmacological support. Conversely, 38 (48.7\%) and 10 (12.8\%) reported sexual function recovery with the use of oral support and/or PGEl administration, respectively, while 18 patients $(23.1 \%)$ reported complete absence of erections. The median IIEF-5 in 29 patients with VA (Group B+D) was 14 (IQR 7-18) while was 22 (IQR 17-24) in 49 nonVA patients (Group A+C) (62.8\%), with a statistically significant difference between the two groups $(p<0.001)$ (Figure 3). Twenty-three patients (79.3\%) with VA reported complete continence, while 6 (20.7\%) used $\geq 2$ pads per day. On the other hand, 48 patients (97.9\%) without VA reported complete continence. All follow-up data stratified for body habitus groups have been showed in Table 2.

\section{Multivariate analysis findings}

At multivariable analysis, visceral adiposity was confirmed as a strong independent predictor for worse continence (Group B: HR 3.67; 2.75-4.51 CI 95\%; p = 0.003; Group D: HR 2.03; 1.81-3.14 CI 95\%; p = 0.04) and sexual function recovery (Group B: HR 4.51; 3.09-5.63 CI 95\%; $\mathrm{p}=0.001 ;$ Group D: HR 3.33; 3.04-5.09 CI 95\%; p = 0.001) (Table 3).

\section{Discussion}

\section{Main findings}

It is widely known that functional outcomes have a non-negligible impact on health-related quality of life after RP.

The impact of obesity on the outcomes of RP, irrespective of surgical approach, has been extensively investigated but we are still far from drawing definitive conclusions (7). Yet there is a strong need for further investigation to explore association between continence and sexual recovery and obesity, assessed not only by BMI but also evaluating district adiposity parameters such as WC, subcutaneous and abdominal fat volume.

To address this unmet need, we conducted the current longitudinal, cohort study to further pose a little cornerstone towards an in-depth knowledge of this critical issue.

On the basis of this background, we demonstrated that obesity with central adiposity was associated with worse continence and sexual function recovery after laparoscopic RP. 
Table 3.

Multivariate analysis results of factors associated with worse functional outcome (urinary and sexual function).

\begin{tabular}{|c|c|}
\hline Categories (variables) & Multivariate analysis (p) (HR; 95\% Cl) \\
\hline \multicolumn{2}{|l|}{ Urinary function } \\
\hline Age ( $<65, \geq 65$ years) & (0.57) (HR $1.01 ; 0.75-1.34)$ \\
\hline Body Mass Index (kg/m², continuous) & (0.07) (HR 1.62; 0.91-1.92) \\
\hline Waist (cm, continuous) & (0.32) (HR 1.13; 0.55-1.87) \\
\hline \multicolumn{2}{|l|}{ Body habitus } \\
\hline Non-obese & (0.08) (HR 0.95; 0.63-0.99) \\
\hline Non-obese with central adiposity & (0.04) (HR 2.03; 1.81-3.14) \\
\hline Obese without central adiposity & (0.09) (HR 1.19; 0.69-1.90) \\
\hline Obese with central adiposity & (0.003) (HR 3.67; 2.75-4.51) \\
\hline Charlson Comorbidity Index $(0-1,>2)$ & (0.89) (HR 1.12; 0.70-1.56) \\
\hline American Society of Anesthesiologists Score $(\leq 2, \geq 3)$ & (0.09) (HR 1.33; 0.90-1.60) \\
\hline \multicolumn{2}{|l|}{ Sexual function } \\
\hline Age $(<65, \geq 65$ years $)$ & (0.63) (HR 1.07; 0.43-1.65) \\
\hline Body Mass Index (kg/m², continuous) & (0.11) (HR 1.82; 0.90-1.96) \\
\hline Waist (cm, continuous) & (0.28) (HR 0.91; 0.34-1.23) \\
\hline \multicolumn{2}{|l|}{ Body habitus } \\
\hline Non-obese & (0.12) (HR 0.80; 0.58-1.12) \\
\hline Non-obese with central adiposity & (0.001) (HR 3.33; 3.04-5.09) \\
\hline Obese without central adiposity & (0.08) (HR 1.20; 0.71-1.87) \\
\hline Obese with central adiposity & (0.001) (HR 4.51; 3.09-5.63) \\
\hline Charlson Comorbidity Index $(0-1,>2)$ & (0.77) (HR 1.93; 0.77-1.60) \\
\hline American Society of Anesthesiologists Score $(\leq 2, \geq 3)$ & (0.93) (HR 1.42; 0.84-1.79) \\
\hline
\end{tabular}

\section{Results in the context of previous studies}

First key point of our study is that visceral obesity was confirmed to be independently associated with worse sexual function recovery. Of course, the presence of a greater amount of periprostatic adipose tissue may be associated with a higher risk of injury to the neurovascular bundle. Moreover, metabolic syndrome itself is linked with worse potency and higher rates of endothelial disfunction (21). Actually, several previous studies showed no impact of obesity on sexual domain after open and/or robotic RP (22-24), while other series reported adverse effects (25) or impact with the metabolic and systemic disease $(26,27)$. However, we would like to point out that in all the above-mentioned health-related quality of life studies, the definition of potency and its measurement was mostly subjective, meaningfully undermining reliability of reported finding. In our study, we tried to overcome this limit by objectively defining pre- and postoperative erectile function with IIEF-5 questionnaire. Second, visceral obesity resulted an independent predictor also of delayed continence recovery. Consistently with our findings, Wiltz et al. (25) published one of the largest series, with 945 patients stratified according to BMI, reporting that obesity was associated with worse continence recovery at 12 and 24 months (25). Moreover, a systematic review and metanalysis by Xu et al. confirmed that obese patients are at higher risk of experiencing worse functional outcomes after RP (28). Of course, obesity might also bring about additional physical strain on the bladder, ultimately resulting in more preoperative urinary problems and a prolonged duration of return to continence. Considering these underlying issues unrelat- ed to surgical expertise, suboptimal functional outcomes should be discussed with obese patients during preoperative counseling.

\section{Strengths and limitations of this study}

The present study was not devoid of limitations. First, this was a retrospective review of a prospectively collected database. Second, the relatively small sample size together might have undermined the evaluation of potential predictors of functional outcomes in our series. Even if all cases were performed by a single surgeon with extensive experience in LRP, our findings could not be applicable to all surgeon- or center-related scenarios. Acknowledged these limitations, our study represents the largest series so far exploring association between continence and sexual recovery and obesity, assessed not only by BMI but also evaluating district adiposity parameters such as WC, subcutaneous and abdominal fat volume. Further multi-institutional series are warranted to confirm our preliminary findings.

\section{Conclusions}

In our experience, visceral adiposity was associated with worse continence and sexual function recovery after laparoscopic RP, highlighting the need for an accurate pre-surgical evaluation of the body habitus and a detailed preoperative counselling before surgery.

\section{REFERENCES}

1. Mottet N, van den Bergh RCN, Briers E, et al. EAU-EANMESTRO-ESUR-SIOG Guidelines on prostate cancer-2020 Update. Part 1: Screening, diagnosis, and local treatment with curative intent. Eur Urol. 2021; 79:243-262.

2. Perletti G, Magri V, Vral A, et al. Green tea catechins for chemoprevention of prostate cancer in patients with histologically-proven HG-PIN or ASAP. Concise review and meta-analysis. Arch Ital Urol Androl. 2019; 91:153-156

3. Sanda MG, Dunn RL, Michalski J, et al. Quality of life and satisfaction with outcome among prostate-cancer survivors. N Engl J Med. 2008; 358:1250-61.

4. Neumaier MF, Segall Júnior $\mathrm{CH}$, Hisano $\mathrm{M}$, et al. Factors affecting urinary continence and sexual potency recovery after roboticassisted radical prostatectomy. Int Braz J Urol. 2019; 45:703-712.

5. Briganti A, Gallina A, Suardi N, et al. Predicting erectile function recovery after bilateral nerve sparing radical prostatectomy: a proposal of a novel preoperative risk stratification. J Sex Med. 2010; 7:2521-31.

6. Lindner U, Lawrentschuk N, Abouassaly R, et al. Radical prostatectomy in obese patients: Improved surgical outcomes in recent years. Int J Urol. 2010; 17:727-32.

7. Yu YD, Byun SS, Lee SE, et al. Impact of body mass index on oncological outcomes of prostate cancer patients after radical prostatectomy. Sci Rep. 2018; 8:11962.

8. Khoder WY, Trottmann M, Stuber A, et al. Early incontinence after radical prostatectomy: A community based retrospective analysis in 911 men and implications for preoperative counseling. Urol Oncol. 2013; 31:1006-11.

9. Luciani LG, Mattevi D, Mantovani W, et al. Retropubic, laparo- 
scopic, and robot-assisted radical prostatectomy: a comparative analysis of the surgical outcomes in a single regional center. Curr Urol. 2017; 11:36-41.

10. Van Velthoven RF, Ahlering TE, Skarecky DW, et al. Technique for laparoscopic running urethrovesical anastomosis: the single knot method. Urology. 2003; 61: 699-702.

11. Ludovico GM, Dachille G, Pagliarulo $G$, et al. Bilateral nerve sparing robotic-assisted radical prostatectomy is associated with faster continence recovery but not with erectile function recovery compared with retropubic open prostatectomy: the need for accurate selection of patients. Oncol Rep. 2013; 29:2445-50.

12. D'Amico AV, Whittington R, Malkowicz SB, et al. Biochemical outcome after radical prostatectomy, external beam radiation therapy, or interstitial radiation therapy for clinically localized prostate cancer. JAMA. 1998; 280:969-74.

13. Kassi E, Pervanidou P, Kaltsas G, et al. Metabolic syndrome: Definitions and controversies. BMC Med. 2011; 9:48.

14. De Nunzio C, Albisinni S, Freedland SJ, et al. Abdominal obesity as risk factor for prostate cancer diagnosis and high grade disease: a prospective multicenter Italian cohort study. Urol Oncol. 2013; 31:9971002

15. Amato MC, Giordano C, Galia M, et al. Visceral Adiposity Index: A reliable indicator of visceral fat function associated with cardiometabolic risk. Diabetes Care. 2010; 33:920-2.

16. Wei J, Liu X, Xue H, et al. Comparisons of visceral adiposity index, body shape index, body mass index and waist circumference and their associations with diabetes mellitus in adults. Nutrients. 2019; 11:1580.

17. Cai $T$, Nesi $G$, Tinacci $G$, et al. Clinical importance of lymph node density in predicting outcome of prostate cancer patients. J Surg Res. 2011; 167:267-72.

18. Cappelleri JC, Rosen RC, Smith MD, et al. Diagnostic evaluation of the erectile function domain of the International Index of Erectile Function. Urology 2009; 54:346-351.
19. Badia X, Garcia-Losa M, Dal-Re R. Ten-language translation and harmonization of the International Prostate Symptom Score: developing a methodology for multinational clinical trials. Eur Urol. 1997; 31:129-40.

20. Palmieri A, Arcaniolo D, Palumbo F, et al. SIA-Low intensity shock wave for Erectile Dysfunction (LED) Study Group. Low intensity shockwave therapy in combination with phosphodiesterase-5 inhibitors is an effective and safe treatment option in patients with vasculogenic ED who are PDE5i non-responders: a multicenter single-arm clinical trial. Int J Impot Res. 2020 Jul 18. doi: 10.1038/s41443-020-0332-7. Epub ahead of print.

21. Otunctemur A, Ozbek E, Cakir SS, et al. Association of erectile dysfunction and urolithiasis. Arch Ital Urol Androl. 2014; 86:215-6.

22. Freedland SJ, Haffner MC, Landis PK, et al. Obesity does not adversely affect health-related quality-of-life outcomes after anatomic retropubic radical prostatectomy. Urology. 2005; 65:1131-6.

23. Uffort EE, Jensen JC. Impact of obesity on early erectile function recovery after robotic radical prostatectomy. JSLS. 2011; 15:32-7.

24. Garg T, Young AJ, Kost KA, et al. Patient-reported quality of life recovery curves after robotic prostatectomy are similar across body mass index categories. Investig Clin Urol. 2017; 58:331-338.

25. Wiltz AL, Shikanov S, Eggener SE, et al. Robotic Radical Prostatectomy in Overweight and Obese Patients: Oncological and Validated-Functional Outcomes. Urology. 2009; 73:316-22.

26. Antunes HP, Teixo R, Carvalho JA, et al. Diabetes mellitus and prostate cancer metabolism: Is there a relationship? Arch Ital Urol Androl. 2018; 90:184-190.

27. Parazzini F, Artibani W, Carrieri G, et al. Effect of body mass and physical activity at younger age on the risk of prostatic enlargement and erectile dysfunction: Results from the 2018 \#Controllati survey. Arch Ital Urol Androl. 2020; 91:245-250.

28. Xu T, Wang X, Xia L, et al. Robot-assisted prostatectomy in obese patients: How influential is obesity on operative outcomes? J Endourol. 2015; 29:198-208.

\section{Correspondence}

Tommaso Cai, MD

ktommy@libero.it

Stefano Chiodini, MD

Lorenzo Giuseppe Luciani, MD

Gianni Malossini, MD

Department of Urology, Santa Chiara Regional Hospital

Largo Medaglie d'Oro, 9, Trento (Italy)

Andrea Cocci, MD

Fabrizio Di Maida, MD

Department of Urology, University of Florence, Florence (Italy)

Francesco Ciarleglio, MD

Department of Surgery, Santa Chiara Regional Hospital, Trento, Italy

Giovanni Pedrotti, MD

Department of Anesthesiology, Santa Maria del Carmine Hospital, Rovereto (Italy)

Alessandro Palmieri, MD

Department of Urology, University Federico II, Naples (Italy)

Michele Rizzo, MD

Giovanni Liguori, MD

Department of Urology, University of Trieste, Trieste (Italy)

Truls E. Bjerklund Johansen, MD

Department of Urology, Oslo University Hospital, Oslo (Norway) 